\title{
Energetics of the layer-thickness form drag based on an integral identity
}

\author{
H. Aiki ${ }^{1}$ and T. Yamagata ${ }^{1,2}$ \\ ${ }^{1}$ Frontier Research Center for Global Change, Japan Agency for Marine-Earth Science and Technology, Yokohama-city \\ 236-0001, Japan \\ ${ }^{2}$ Department of Earth and Planetary Science, Graduate School of Science, University of Tokyo, Tokyo 113-0033, Japan
}

Received: 18 April 2006 - Published in Ocean Sci. Discuss.: 20 June 2006

Revised: 25 September 2006 - Accepted: 9 October 2006 - Published: 12 October 2006

\begin{abstract}
The vertical redistribution of the geostrophic momentum by the residual effects of pressure perturbations (called the layer-thickness form drag) is investigated using thickness-weighted temporal-averaged mean primitive equations for a continuously stratified fluid in an adiabatic formulation. A four-box energy diagram, in which the mean and eddy kinetic energies are defined by the thickness-weighted mean velocity and the deviation from it, respectively, shows that the layer-thickness form drag reduces the mean kinetic energy and endows the eddy field with an energy cascade. The energy equations are derived using an identity (called the "pile-up rule") between cumulative sums of the Eulerian mean quantity and the thickness-weighted mean quantity in each vertical column. The pile-up rule shows that the thickness-weighted mean velocity satisfies a no-normal-flow boundary condition at the top and bottom of the ocean, which enables the volume budget of pressure flux divergence in the energy diagram to be determined. With the pile-up rule, the total kinetic energy based on the Eulerian mean can be rewritten in a thickness-weighted form. The four-box energy diagram in the present study should be consistent with energy diagrams of layer models, the temporal-residual-mean theory, and Iwasaki's atmospheric theory. Under certain assumptions, the work of the layer-thickness form drag in the global ocean circulation is suggested to be comparable to the work done by the wind forcing.
\end{abstract}

\section{Introduction}

The vertical mixing of momentum in a stratified fluid can be induced by the residual effects of pressure perturbations (called the layer-thickness form drag in this paper, as detailed

Correspondence to: $\mathrm{H}$. Aiki

(aiki@jamstec.go.jp) in Sect. 2.1), which has received considerable attentions in various research areas of atmosphere and ocean dynamics (cf. Andrews, 1983; Johnson and Bryden, 1989; CushmanRoisin et al., 1990; Lee and Leach, 1996). In contrast to the momentum transfer, energetics of the layer-thickness form drag have received little attention in previous oceanic studies. The present study shows that an adiabatic formulation of an inviscid hydrostatic fluid yields a four-box energy diagram that elucidates the role of layer-thickness form drag in the connection between the mean and perturbation fields. This result can be regarded as fundament to introducing the parameterization of layer-thickness form drag in numerical ocean circulation models.

The four-box energy diagram of ocean and atmosphere dynamics consists of the potential and kinetic energies associated with the mean and perturbation fields. The classical Lorenz (1955) diagram has often been used in the theoretical development of subgrid-scale parameterization in numerical simulations and in the analysis of various types of data (Böning and Budich, 1992; Holton, 1992). However, the energy diagram and associated energy cycle may vary with the definitions of the mean and perturbation fields. An energy diagram for the transformed Eulerian mean (TEM) theory (Andrews and McIntyre, 1976) is given by Plumb (1983) and Kanzawa (1984), whereas an energy diagram for the generalized Lagrangian mean (GLM) theory (Andrews and McIntyre, 1978) has received little attention in past oceanic studies. Focusing on the adiabatic aspects of waves and eddies in a stratified fluid, Iwasaki (2001) derived a new energy diagram from a one-dimensional (vertical direction) analog of the GLM. He showed that the layer-thickness form drag allows direct transfer between the mean kinetic and eddy potential energies, which replaces the route involving the eddy kinetic energy in the TEM theory. Moreover, Iwasaki's formulation does not use the geostrophic balance in closing the

Published by Copernicus GmbH on behalf of the European Geosciences Union. 


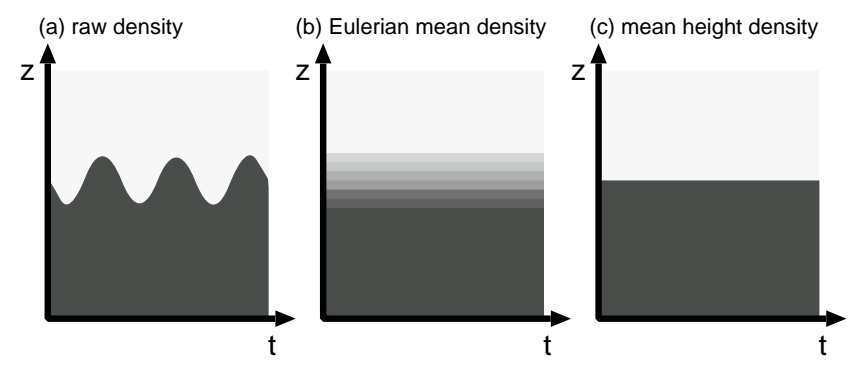

Fig. 1. Views of (a) the raw density $\rho(z, t)$, showing the vertical fluctuation of a density surface in a two-density fluid; (b) the Eulerian mean density $\bar{\rho}(z, t)$, which is given by the fixed-height temporal average; and (c) the mean height density $\widetilde{\rho}(z, t)$, which is a z-coordinate expression of the adiabatically low-pass filtered layer interface. A darker shade indicates higher density.

energy diagram, which is in sharp contrast to the situation with the TEM theory. This allows Iwasaki's energy diagram to be applied various types of (rotational and nonrotational) stratified fluids. The result of Iwasaki (2001) follows that of Bleck (1985), who showed that the mean and eddy kinetic energies can be positive-definite quantities in isentropic coordinates.

The present study investigated the characteristics of Iwasaki's energy diagram in order to clarify the role of layerthickness form drag in the connection between the mean and perturbation fields, with the aim of understanding the effects of introducing layer-thickness form drag in coarse-resolution ocean models (cf. Greatbatch, 1998), as part of parameterization of unresolved geostrophic eddies in baroclinic instability (Charney, 1947; Eady, 1949). In order to elucidate the components required in the new energy diagram, this paper does not use the semi-Lagrangian coordinates of Andrews and McIntyre (1978), Iwasaki (2001), and Jacobson and Aiki (2006). The present derivation begins with the inviscid incompressible hydrostatic Boussinesq equations, which are adiabatically low-pass filtered so as to avoid unphysical mixing across density surfaces. These equations are essentially the thickness-weighted-mean equations (for tracers, density, and momentum) in density-coordinates (de Szoeke and Bennett, 1993), as explained in Sect. 2. We focus on an integral identity to explain the boundary condition (Sect. 2.2). In Sect. 3, we present an energy diagram for the above adiabatically low-pass filtered equations which is largely consistent with the work of Iwasaki (2001). Under certain assumptions on form-drag parameterization, the work associated with the eddy form drag in the global ocean circulation is estimated in Sect. 4. The paper concludes with a summary in Sect. 5. The present study excluded diabatic processes (density mixing) in the surface mixed layer and the bottom boundary layer of the ocean (cf. Kuo et al., 2005; Plumb and Ferrari, 2005), since we are concerned with the adiabatic process (mesoscale ed- dies) and the boundary condition of the present formulation is clear, in contrast to the TEM theory (see Sects. 2.2 and 3.5).

\section{Adiabatic mean formulation}

Section 2.1 summarizes the thickness-weighted temporalmean momentum and density equations that have been investigated by de Szoeke and Bennett (1993), McDougall and McIntosh (2001), and Jacobson and Aiki (2006). Readers not familiar with expressions in Sect. 2.1 are first referred to Bleck (1985) for the primitive equations (and energy equations) in isentropic and density coordinates. In Sect. 2.2, we introduce an integral identity to explain the boundary condition.

\subsection{Primitive equations}

The parameterization of mesoscale eddy transports with an additional advection represented a major advance in ocean modeling, that allowed coarse-resolution models to maintain deep water formation in the polar regions and overturning circulation in the world's oceans (Danabasoglu and McWilliams, 1994; Gent et al., 1995; Treguier et al., 1997; Killworth, 1997). These theories are based on the thicknessweighted-mean formulation of a passive tracer equation in density-coordinates (note that "averaging" refers to a temporal low-pass filter in this paper). De Szoeke and Bennett (1993) pointed out in their Appendix A that the mean quantities in density-coordinates can be mapped back onto zcoordinates (i.e., Cartesian coordinates). That is, a thicknessweighted-mean $\overline{((\partial z / \partial \rho) S)}^{\rho} / \overline{(\partial z / \partial \rho)}^{\rho}$ is taken in densitycoordinates (where $S$ is an arbitrary quantity and $\rho$ is density), which is then mapped back into z-coordinates that now refer to the mean vertical position of each isopycnal surface. This backmapped quantity, now a function of $z$, is here given the symbol $\widehat{S}$ (Table 1 describes the symbols used in this paper). A passive tracer equation such as $\partial S / \partial t+\boldsymbol{U} \cdot \nabla S=0$ in $\mathbf{z}$-coordinates becomes, after one application of this process, $\partial \widehat{S} / \partial t+\widehat{\boldsymbol{U}} \cdot \nabla \widehat{S}=M[S]$, where $\widehat{\boldsymbol{U}}$ is the thickness-weighted three-dimensional velocity in mean z-coordinates and $M[]$ is the isopycnal mixing (cf. Griffies, 2004). The weighted three-dimensional velocity is nondivergent $(\nabla \cdot \widehat{\boldsymbol{U}}=0)$ if the unweighted three-dimensional velocity is nondivergent $(\nabla \cdot \boldsymbol{U}=0)$.

In the special case where the density equation is, $M[\rho]=0$, diffusion is not present. In densitycoordinates, the thickness-weighted mean density is $\overline{((\partial z / \partial \rho) \rho)} \rho / \overline{(\partial z / \partial \rho)}^{\rho}=\bar{\rho}^{\rho}=\rho$. As a result, it is useful to introduce $\widetilde{S}$ for an isopycnal mean (but not thicknessweighted) quantity $\bar{S}^{\rho}$ that is backmapped onto z-coordinates at the mean vertical position of each density surface (Ta- 
Table 1. List of symbols, where $S(x, y, \rho, t)$ is an arbitrary quantity.

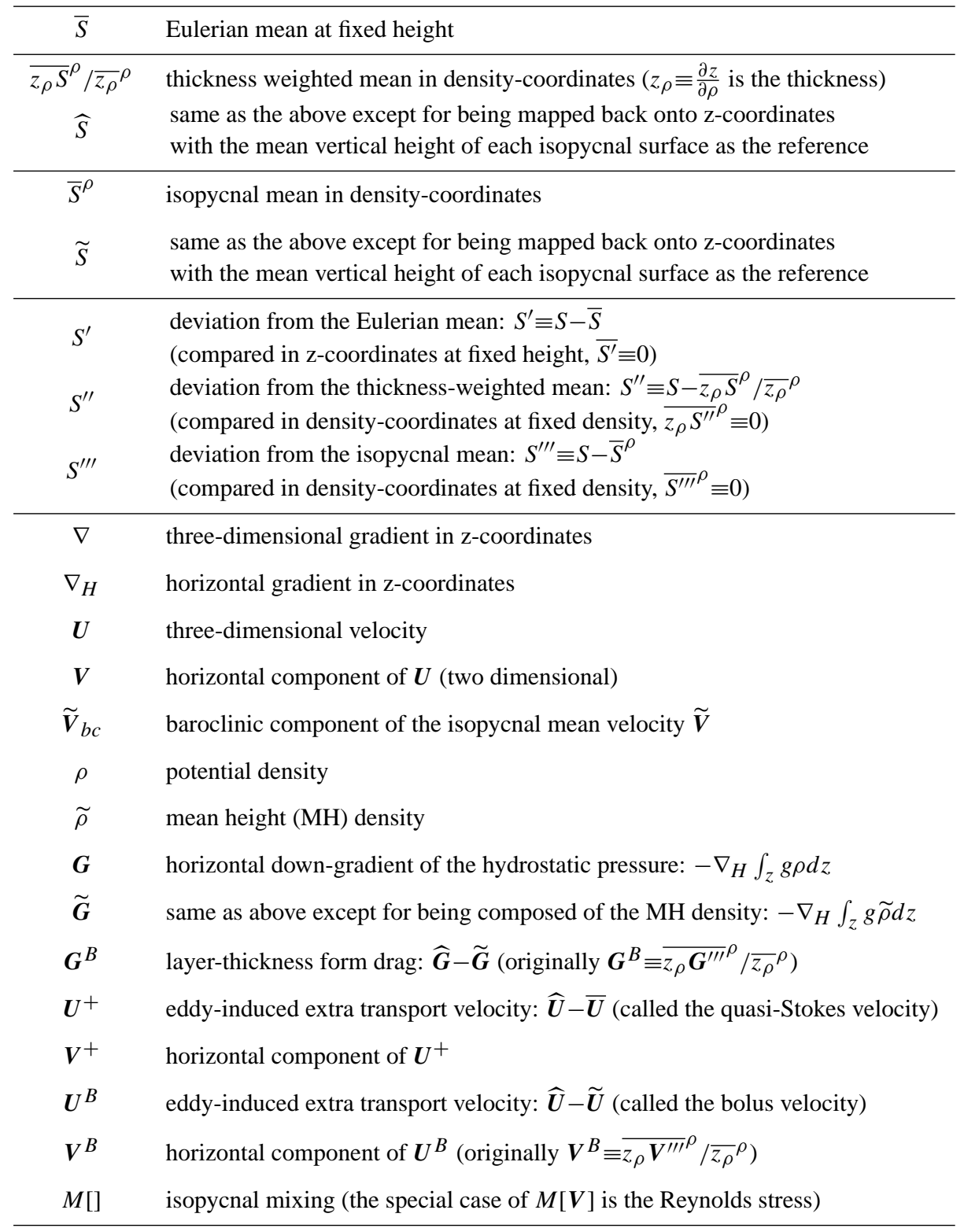

ble 1). The modified density equation (de Szoeke and Bennett, 1993) in z-coordinates becomes

$\frac{\partial}{\partial t} \widetilde{\rho}+\widehat{\boldsymbol{U}} \cdot \nabla \widetilde{\rho}=0$.

Here we call $\tilde{\rho}$ the mean height $(\mathrm{MH})$ density: this is the same as the temporal-residual-mean (TRM) density in McDougall and McIntosh (2001) and Jacobson and Aiki (2006), and is given by the density of the surface whose mean vertical position is $z$. Stratification of the MH density $\widetilde{\rho}$ is inherently sharper than that of the Eulerian mean density $\bar{\rho}$ (see Fig. 1; a related discussion appears in Killworth, 2001). In most ocean general circulation models (OGCMs), the thickness- weighted velocity $\widehat{\boldsymbol{U}}$ to advect tracers is calculated by summing the prognostic velocity in the model and a parameterized extra transport velocity (detailed in Sect. 3.3), because in the prevailing mean formulations the momentum equations are simply averaged either by the isopycnal mean (Gent et al., 1995) or the Eulerian mean (McDougall and McIntosh, 1996) to avoid modifying the form of the pressure term.

However, an interesting feature appears when the momentum equations are also thickness weighted: the hydrostatic pressure gradients $-\nabla_{H} \int_{z} g \rho d z(\equiv \boldsymbol{G})$ yield a secondary term $G^{B}$ (i.e., the layer-thickness form drag, eddy form drag, or inviscid pressure drag) in addition to the term available to 
the model $-\nabla_{H} \int_{z} g \widetilde{\rho} d z(\equiv \widetilde{\boldsymbol{G}})$, where $\nabla_{H}=(\partial / \partial x, \partial / \partial y)$. Table 1 provides a detailed expression of $\boldsymbol{G}^{B}(\equiv \widehat{\boldsymbol{G}}-\widetilde{\boldsymbol{G}})$. The thickness-weighted mean momentum equation is

$$
\frac{\partial}{\partial t} \widehat{\boldsymbol{V}}+\widehat{\boldsymbol{U}} \cdot \nabla \widehat{\boldsymbol{V}}+f \boldsymbol{z} \times \widehat{\boldsymbol{V}}=\widetilde{\boldsymbol{G}} / \rho_{0}+\boldsymbol{G}^{B} / \rho_{0}+M[\boldsymbol{V}],
$$

where $\widehat{\boldsymbol{V}}=(\widehat{u}, \widehat{v})$ and $f$ is the Coriolis parameter of the earth. The Reynolds stress $M[V]$ is less focused on in the present paper, and the total transport velocity $\widehat{\boldsymbol{U}}$ has no component normal to solid boundaries (McDougall and McIntosh, 2001; see Sect. 2.2 for details).

Equations (1) and (2) first appeared in de Szoeke and Bennett (1993) in an adiabatic and macroscopic context, and were further investigated in later studies. McDougall and McIntosh (2001) introduced a Taylor expansion for the vertical displacement of density surfaces relative to z-coordinates. To present exact equations for the mean and perturbation fields, Jacobson and Aiki (2006) used a height-density semiLagrangian coordinate that is analogous to the pressureisentrope semi-Lagrangian coordinate of Iwasaki (2001). Equations (1) and (2) are now well justified, being free from expansion parameters and the explicit use of densitycoordinates, which are improvements over McDougall and McIntosh (2001) and de Szoeke and Bennett (1993), respectively. Most importantly, velocity $\widehat{\boldsymbol{U}}$ is a prognostic quantity in a model stepping forward Eqs. (1) and (2), and this suggests the applicability of a momentum approach in which the pressure drag $\boldsymbol{G}^{B}$ rather than the eddy-induced advection is parameterized (Greatbatch, 1998; Ferreira et al., 2005).

\subsection{Boundary condition}

We consider an oceanic domain bounded by a rigid sea surface and a bottom with arbitrary topography. To show that the total transport velocity $\widehat{\boldsymbol{U}}$ has no component normal to the boundaries, we here introduce an identity for the vertical integrals of Eulerian mean and thickness-weighted mean quantities:

$$
\begin{aligned}
& \int_{-h}^{0} \bar{S} d z=\overline{\int_{-h}^{0} S d z}=\overline{\int_{\rho_{b t m}}^{\rho_{\mathrm{top}}}\left(S \frac{\partial z}{\partial \rho}\right) d \rho}= \\
& \int_{\widetilde{\rho}_{b t m}}^{\widetilde{\rho}_{\mathrm{top}}} \overline{\left(S \frac{\partial z}{\partial \rho}\right)^{\rho}} d \widetilde{\rho}=\int_{-h}^{0} \frac{\overline{\left(S \frac{\partial z}{\partial \rho}\right)}}{\partial z / \partial \widetilde{\rho}} d z=\int_{-h}^{0} \widehat{S} d z,
\end{aligned}
$$

where $h(>0)$ is the bottom depth. This identity, which applies to any quantity $S$, is a generalization of the results of McDougall and McIntosh (2001) and Killworth (2001), and is here called the "pile-up rule" since it explains the relations between the cumulative sums of weighted differentials in the vertical direction. An obvious interpretation of the pile-up rule is that, with $T$ denoting the range of time averaging, both $T \int_{-h}^{0} \bar{S} d z$ and $T \int_{-h}^{0} \widehat{S} d z$ refer to a net amount $\int_{t-T / 2}^{t+T / 2} \int_{-h}^{0} S d z d t$ in $(z, t)$ space, measured with zcoordinates and density-coordinates, respectively.

Because the no-normal-flow condition of the Eulerian mean velocity $\overline{\boldsymbol{U}}$ is obvious, it is essential to show how the remaining part $\widehat{\boldsymbol{U}}-\overline{\boldsymbol{U}}\left(=\boldsymbol{U}^{+}\right.$, called the quasi-Stokes velocity in McDougall and McIntosh, 2001) satisfies the boundary condition (Table 1). The pile-up rule, Eq. (3), makes the horizontal component of the quasi-Stokes velocity purely baroclinic: $\int_{-h}^{0} \boldsymbol{V}^{+} d z\left(=\int_{-h}^{0} \widehat{\boldsymbol{V}}-\overline{\boldsymbol{V}} d z\right)=0$. This allows the overturning stream function $\int_{-h}^{z} \boldsymbol{V}^{+} d z\left(=\int_{-h}^{z} \widehat{\boldsymbol{V}}-\overline{\boldsymbol{V}} d z\right)$ to vanish at the top and bottom boundaries, which confirms the no-normal-flow boundary condition of $\boldsymbol{U}^{+}$. As a result, the total transport velocity $\widehat{\boldsymbol{U}}$ has no component normal to the top and bottom boundaries, in sharp contrast to the total transport velocity used in Plumb (1983) and Kanzawa (1984).

Explaining the boundary condition becomes less straightforward when the pile-up rule is not used (Bleck, 1985; Jacobson and Aiki, 2006). The pile-up rule turns out to be useful also for the derivation of energy equations (Sect. 3).

\section{Energy equations}

Both the potential and kinetic energies are subject to temporal low-pass filtering, resulting in the so-called total potential and total kinetic energies, respectively, whose equations for inviscid hydrostatic Boussinesq fluids are

$$
\begin{aligned}
& \frac{\partial}{\partial t} \overline{\rho g z}+\overline{\nabla \cdot(\boldsymbol{U} \rho g z)}=g \overline{w \rho}, \\
& \frac{\partial}{\partial t}\left(\frac{\rho_{0}}{2} \overline{|\boldsymbol{V}|^{2}}\right)+\overline{\nabla \cdot\left(\boldsymbol{U} \frac{\rho_{0}}{2}|\boldsymbol{V}|^{2}\right)}=\overline{\boldsymbol{V} \cdot \boldsymbol{G}},
\end{aligned}
$$

where the overbar denotes the Eulerian temporal mean at a constant height. The energy interaction is determined by the pressure-flux divergence:

$\overline{-\nabla \cdot\left(\boldsymbol{U} \int_{z} \rho g d z\right)}=\overline{\boldsymbol{V} \cdot \boldsymbol{G}}+g \overline{w \rho}$,

which includes the incompressibility condition $\nabla \cdot \boldsymbol{U}=0$. To simplify the problem, we consider a volume integral in a closed domain $\Omega$ with solid boundaries (i.e., rigid sea surface). Because the raw velocity $\boldsymbol{U}$ has no component crossing the boundaries of the domain, the volume integral of Eqs. (4-6) becomes

$$
\begin{aligned}
& \frac{d}{d t} \int_{\Omega} \bar{\rho} g z d^{3} x=g \int_{\Omega} \overline{w \rho} d^{3} x, \\
& \frac{\rho_{0}}{2} \frac{d}{d t} \int_{\Omega} \overline{|\boldsymbol{V}|^{2}} d^{3} x=\int_{\Omega} \overline{\boldsymbol{V} \cdot \boldsymbol{G}} d^{3} x, \\
& 0=\int_{\Omega} \overline{\boldsymbol{V} \cdot \boldsymbol{G}} d^{3} x+\int_{\Omega} g \overline{w \rho} d^{3} x .
\end{aligned}
$$

In the absence of boundary forcing and friction, the sum of the total potential and total kinetic energies is constant. 


\subsection{Mean field}

The component of the total energy that is written in terms of resolved quantities, such as $\widetilde{\rho}$ and $\widehat{\boldsymbol{V}}$, is traditionally called the mean energy (a clearer term is the resolved mean energy). The mean potential and mean kinetic energies and their interaction can be derived from Eqs. (1), (2), and the incompressibility condition $\nabla \cdot \widehat{\boldsymbol{U}}=0$ :

$$
\begin{aligned}
& \frac{\partial}{\partial t}(\widetilde{\rho} g z)+\nabla \cdot(\widehat{\boldsymbol{U}} \tilde{\rho} g z)=g \widehat{\boldsymbol{w}} \tilde{\rho} \\
& \begin{array}{c}
\frac{\partial}{\partial t}\left(\frac{\rho_{0}}{2}|\widehat{\boldsymbol{V}}|^{2}\right)+\nabla \cdot\left(\widehat{\boldsymbol{U}} \frac{\rho_{0}}{2}|\widehat{\boldsymbol{V}}|^{2}\right)= \\
\widehat{\boldsymbol{V}} \cdot\left(\widetilde{\boldsymbol{G}}+\boldsymbol{G}^{B}\right)+\rho_{0} \widehat{\boldsymbol{V}} \cdot M[\boldsymbol{V}], \\
-\nabla \cdot\left(\widehat{\boldsymbol{U}} \int_{z} \tilde{\rho} g d z\right)=\widehat{\boldsymbol{V}} \cdot \widetilde{\boldsymbol{G}}+g \widehat{w} \widetilde{\rho} .
\end{array}
\end{aligned}
$$

The mean kinetic energy in Eq. (11) is defined in terms of the thickness-weighted mean velocity, as in Bleck (1985) and Jacobson and Aiki (2006). The quantity $|\widehat{\boldsymbol{V}}|$ is the magnitude of (the horizontal component of) the total advective velocity including the basic geostrophic current and the eddy-induced overturning transport (detailed in Sect. 3.3). We emphasize that here the mean kinetic energy is defined with neither the Eulerian mean velocity $\overline{\boldsymbol{V}}$ nor the isopycnal mean velocity $\widetilde{\boldsymbol{V}}$. By remembering that the total transport velocity $\widehat{U}$ has no component crossing the boundaries (Sect. 2.2), the volume integral of Eqs. (10-12) becomes

$$
\begin{aligned}
& \frac{d}{d t} \int_{\Omega} \tilde{\rho} g z d^{3} x=g \int_{\Omega} \widehat{w} \tilde{\rho} d^{3} x \\
& \frac{\rho_{0}}{2} \frac{d}{d t} \int_{\Omega}|\widehat{\boldsymbol{V}}|^{2} d^{3} x= \\
& \quad \int_{\Omega} \widehat{\boldsymbol{V}} \cdot \widetilde{\boldsymbol{G}} d^{3} x+\int_{\Omega}\left(\widehat{\boldsymbol{V}} \cdot \boldsymbol{G}^{B}+\rho_{0} \widehat{\boldsymbol{V}} \cdot M[\boldsymbol{V}]\right) d^{3} x \\
& 0=\int_{\Omega} \widehat{\boldsymbol{V}} \cdot \widetilde{\boldsymbol{G}} d^{3} x+\int_{\Omega} g \widehat{w} \tilde{\rho} d^{3} x .
\end{aligned}
$$

The second integral on the right-hand side of Eq. (14) allows transfer of energy between the mean and eddy fields. Both the Reynolds stress $M[\boldsymbol{V}]$ and the layer-thickness form drag $\boldsymbol{G}^{B}$ are responsible for the connection between the mean and eddy fields.

\subsection{Eddy field}

The remaining component of the total energy is traditionally called the eddy energy (a clearer term is the unresolved perturbation energy). Below we derive the eddy energy as the difference between (the residual of) the total and mean energies.
An equation for the eddy potential energy is obtained by subtracting Eq. (10) from Eq. (4), which essentially is the difference between the MH density and the Eulerian mean density. The volume budget of the eddy potential energy is obtained by subtracting Eq. (13) from Eq. (7):

$\frac{d}{d t} \int_{\Omega}(\bar{\rho}-\widetilde{\rho}) g z d^{3} x=g \int_{\Omega}(\overline{\rho w}-\widetilde{\rho} \widehat{w}) d^{3} x$.

It is expected that the energy level associated with $\bar{\rho} g z$ is higher than that of $\tilde{\rho} g z$ because the Eulerian mean density $\bar{\rho}$ is smoothed in the vertical direction (as suggested by Fig. 1). McDougall and McIntosh (2001) showed that the difference between the Eulerian mean density and the $\mathrm{MH}$ density is $\bar{\rho}-\widetilde{\rho}=\left(\phi / \bar{\rho}_{z}\right)_{z}+O\left(\alpha^{3}\right)$, where $\phi \equiv \frac{1}{2} \overline{\rho^{\prime 2}}$ is half the density variance measured at a fixed point in space and $\alpha$ is a scale for density disturbances. Integration by parts yields $\int_{-h}^{0}\left(\phi / \overline{\rho_{z}}\right)_{z} g z d z=-\int_{-h}^{0} \phi g / \bar{\rho}_{z} d z>0$, which is the eddy potential energy referred to in some previous studies. Canuto and Dubovikov (2006), in reviewing the classical energetics of Böning and Budich (1992) and thus Lorenz (1955), presented the same definition of the eddy potential energy. However, if the eddy potential energy is expressed with $\phi$, it involves small errors associated with the Taylor expansion and the top and bottom boundary conditions. The present study uses the exact form of the eddy potential energy, Eq. (16).

An equation for the eddy kinetic energy is obtained by subtracting Eq. (11) from Eq. (5), and the volume budget of the eddy kinetic energy is obtained by subtracting Eq. (14) from Eq. (8). We use the pile-up rule, Eq. (3), to obtain a thickness-weighted form of integral equation for the total kinetic energy, Eq. (8). Equation (8) becomes

$$
\begin{aligned}
\frac{\rho_{0}}{2} \frac{d}{d t} \int_{\Omega}\left(|\widehat{\boldsymbol{V}}|^{2}+\frac{\overline{z_{\rho}\left|\boldsymbol{V}^{\prime \prime}\right|^{2}}}{\overline{z_{\rho}}}\right) d^{3} x= \\
\int_{\Omega}\left(\widehat{\boldsymbol{V}} \cdot \widehat{\boldsymbol{G}}+\frac{\overline{z_{\rho} \boldsymbol{V}^{\prime \prime} \cdot \boldsymbol{G}^{\prime \prime}}}{{\overline{z_{\rho}}}^{\rho}}\right) d^{3} x,
\end{aligned}
$$

where $\boldsymbol{V}^{\prime \prime}=\boldsymbol{V}-{\overline{z_{\rho}}}^{\rho} / \overline{z_{\rho}} \rho$ and $\boldsymbol{G}^{\prime \prime}=\boldsymbol{G}-{\overline{z_{\rho}}}^{\rho} / \bar{z}_{\rho}^{\rho}$ are the deviations from the thickness-weighted mean, in densitycoordinates (see Table 1). Subtracting Eq. (14) from Eq. (17) yields the equation for the eddy kinetic energy:

$$
\begin{aligned}
& \frac{\rho_{0}}{2} \frac{d}{d t} \int_{\Omega} \overline{\left|\boldsymbol{V}^{\prime \prime}\right|^{2}} d^{3} x= \\
& \quad \int_{\Omega} \overline{\boldsymbol{V}^{\prime \prime} \cdot \boldsymbol{G}^{\prime \prime}} d^{3} x-\rho_{0} \int \widehat{\boldsymbol{V}} \cdot M[\boldsymbol{V}] d^{3} x,
\end{aligned}
$$

where we used the pile-up rule, Eq. (3), again for the primed products. The left-hand side of Eq. (18) shows that the eddy kinetic energy is a positive-definite quantity.

An equation for the pressure-flux divergence in the eddy field is obtained by subtracting Eq. (12) from Eq. (6), and the volume budget of the pressure-flux divergence in the eddy 
field is obtained by subtracting Eq. (15) from Eq. (9). We use the pile-up rule, Eq. (3), to obtain a thickness-weighted form of integral Eq. (9), for the energy interaction in the total field. Equation (9) becomes

$$
\begin{aligned}
0 & =\int_{\Omega} \overline{\boldsymbol{V} \cdot \boldsymbol{G}} d^{3} x+\int_{\Omega} g \overline{w \rho} d^{3} x \\
& =\int_{\Omega}\left(\widehat{\boldsymbol{V}} \cdot \widehat{\boldsymbol{G}}+\overline{\boldsymbol{V}^{\prime \prime} \cdot \boldsymbol{G}^{\prime \prime}}\right) d^{3} x+\int_{\Omega} g \overline{w \rho} d^{3} x,
\end{aligned}
$$

where we have used the pile-up rule, Eq. (3), to transform $\overline{\boldsymbol{V} \cdot \boldsymbol{G}}$. Subtracting Eq. (15) from Eq. (19) yields

$0=\int_{\Omega}\left(\widehat{\boldsymbol{V}} \cdot \boldsymbol{G}^{B}+\overline{\boldsymbol{V}^{\prime \prime} \cdot \boldsymbol{G}^{\prime \prime}}\right) d^{3} x+\int_{\Omega} g(\overline{w \rho}-\widehat{w} \widetilde{\rho}) d^{3} x \cdot(20)$

Equations (15) and (20) are used in Sect. 3.4 to close the four-box energy diagram (Fig. 2).

At this point we have a complete set of equations for the mean and eddy energies.

\subsection{Eddy transports}

Before examining the energy diagram, we briefly discuss a separation of the total advective velocity into $\widehat{\boldsymbol{U}} \equiv \widetilde{\boldsymbol{U}}+\boldsymbol{U}^{B}$ (Table 1). The isopycnal mean velocity $\widetilde{\boldsymbol{U}}$ is usually almost in geostrophic balance, and the secondary velocity $U^{B}$ is an eddy-induced overturning circulation that is often called the bolus velocity (Rhines, 1982). The horizontal component of the bolus velocity was originally defined as $\boldsymbol{V}^{B} \equiv{\overline{\left((\partial z / \partial \rho) \boldsymbol{V}^{\prime \prime \prime}\right)}}^{\rho} / \overline{(\partial z / \partial \rho)}^{\rho}$ in layered models, where $\boldsymbol{V}^{\prime \prime \prime} \equiv \boldsymbol{V}-\overline{\boldsymbol{V}}^{\rho}$ is the deviation in density-coordinates from the isopycnal mean (see Table 1). Using the nondivergence of $\widehat{\boldsymbol{U}}$, the vertical component of the bolus velocity in z-coordinates is given by $w^{B}=-\widetilde{w}-\nabla_{H} \cdot \int_{-h}^{z} \widehat{\boldsymbol{V}} d z$. Note that both the bolus velocity and the isopycnal mean velocity are threedimensionally divergent (McDougall, 1998).

By noting $\boldsymbol{V}^{\prime \prime} \equiv \boldsymbol{V}^{\prime \prime \prime}-\boldsymbol{V}^{B}$ in density-coordinates, the eddy kinetic energy in Eq. (18) can be rewritten using

$$
\begin{aligned}
& \int_{-h}^{0} \overline{\left|\boldsymbol{V}^{\prime \prime}\right|^{2}} d z=\int_{-h}^{0} \frac{{\overline{z_{\rho}\left|\boldsymbol{V}^{\prime \prime}\right|^{2}}}^{\rho}}{{\overline{z_{\rho}}}^{\rho}} d z \\
& =\int_{-h}^{0} \frac{\overline{z_{\rho}\left(\left|\boldsymbol{V}^{\prime \prime \prime}\right|^{2}-2 \boldsymbol{V}^{\prime \prime \prime} \cdot \boldsymbol{V}^{B}+\left|\boldsymbol{V}^{B}\right|^{2}\right)^{\rho}}}{{\overline{z_{\rho}}}^{\rho}} d z \\
& =\int_{-h}^{0} \frac{\overline{z_{\rho}\left|\boldsymbol{V}^{\prime \prime \prime}\right|^{2}}}{\overline{z_{\rho}} \rho}-2 \frac{\overline{z_{\rho} \boldsymbol{V}^{\prime \prime \prime}}{ }^{\rho} \cdot \boldsymbol{V}^{B}}{\overline{z_{\rho}} \rho}+\left|\boldsymbol{V}^{B}\right|^{2} d z \\
& =\int_{-h}^{0} \overline{\left|\boldsymbol{V}^{\prime \prime \prime}\right|^{2}}-2\left|\boldsymbol{V}^{B}\right|^{2}+\left|\boldsymbol{V}^{B}\right|^{2} d z \\
& =\int_{-h}^{0} \overline{\left|\boldsymbol{V}^{\prime \prime \prime}\right|^{2}}-\left|\boldsymbol{V}^{B}\right|^{2} d z,
\end{aligned}
$$

where the pile-up rule, Eq. (3), has been applied. Interestingly, Eq. (21) shows that the eddy kinetic energy is a conventional disturbance energy $\left(\rho_{0} / 2\right) \overline{\left|\boldsymbol{V}^{\prime \prime \prime}\right|^{2}}$ (based on the deviation from the isopycnal mean) minus the eddy transport component $\left(\rho_{0} / 2\right)\left|\boldsymbol{V}^{B}\right|^{2}$. It should be noted that velocity $V^{B}$ of the eddy-induced overturning has been included in the mean kinetic energy, $\left(\rho_{0} / 2\right)|\widehat{\boldsymbol{V}}|^{2}$, in the present definition.

\subsection{Energy cycle}

An energy diagram for Eqs. (13), (14), (16), and (18) is illustrated in Fig. 2. In order to elucidate the form of the energy cycle, equations for the energy interaction, Eqs. (15) and (20), are here made independent of the budget of the potential and kinetic energies.

We are primarily concerned with the case of baroclinic instability (Charney, 1947; Eady, 1949), which is a cascade that originates in the mean potential energy in the absence of mechanical forcing at the boundaries. The eddy-induced overturning is essential to relax the slope of density surfaces, which leads to the extraction of the mean potential energy as expressed by $\boldsymbol{V}^{B} \cdot \widetilde{\boldsymbol{G}}$ in Fig. 2. This channel is found to be in the resolved mean field, and it provides an input to the mean kinetic energy (i.e., acceleration of the mean current), in contrast to the classical Lorenz energy diagram (Lorenz, 1955; Böning and Budish, 1992). It is also noted in Fig. 2 that the mean kinetic energy $\left(\rho_{0} / 2\right)|\widehat{\boldsymbol{V}}|^{2}$ will leak to the unresolved perturbation field by $\widehat{\boldsymbol{V}} \cdot \boldsymbol{G}^{B}$ : this is the redistribution of momentum by the form drag.

In the unresolved field in Fig. 2, the quantity $\widehat{\boldsymbol{V}} \cdot \boldsymbol{G}^{B}$ is independently connected to both the eddy potential and kinetic energies; this is due to Eq. (20). In particular, the direct connection between the eddy potential and the mean kinetic energies involves both the density surface perturbation and the layer-thickness form drag. The situation in the unresolved perturbation field is consistent with the result of Iwasaki (2001) derived from the mass-weighted-mean equations for non-Boussinesq fluids. The backmapping method of de Szoeke and Bennett (1993) based on the mean height of each isopycnal surface is consistent with the theory of Iwasaki (2001) based on the mean pressure along each isentropic surface (cf. Kushner and Held, 1999; Greatbatch and McDougall, 2003).

In addition to the form drag, the Reynolds stress $M[V]$ also connects the mean and eddy kinetic energies, which is relevant to the role of relative vorticity in baroclinic instability.

\subsection{Comparison with the TEM and Lorenz formulations}

The present diagram (and that of Iwasaki) may be considered to be partly analogous to the TEM energy diagram (Plumb, 1983; Kanzawa, 1984), in that the fraction of the mean potential energy extracted by the eddy-induced overturning is able to interact with the mean kinetic energy before cascading to the unresolved field. The TEM energy diagram differs from that of Lorenz (1955) not because of a different definition of 


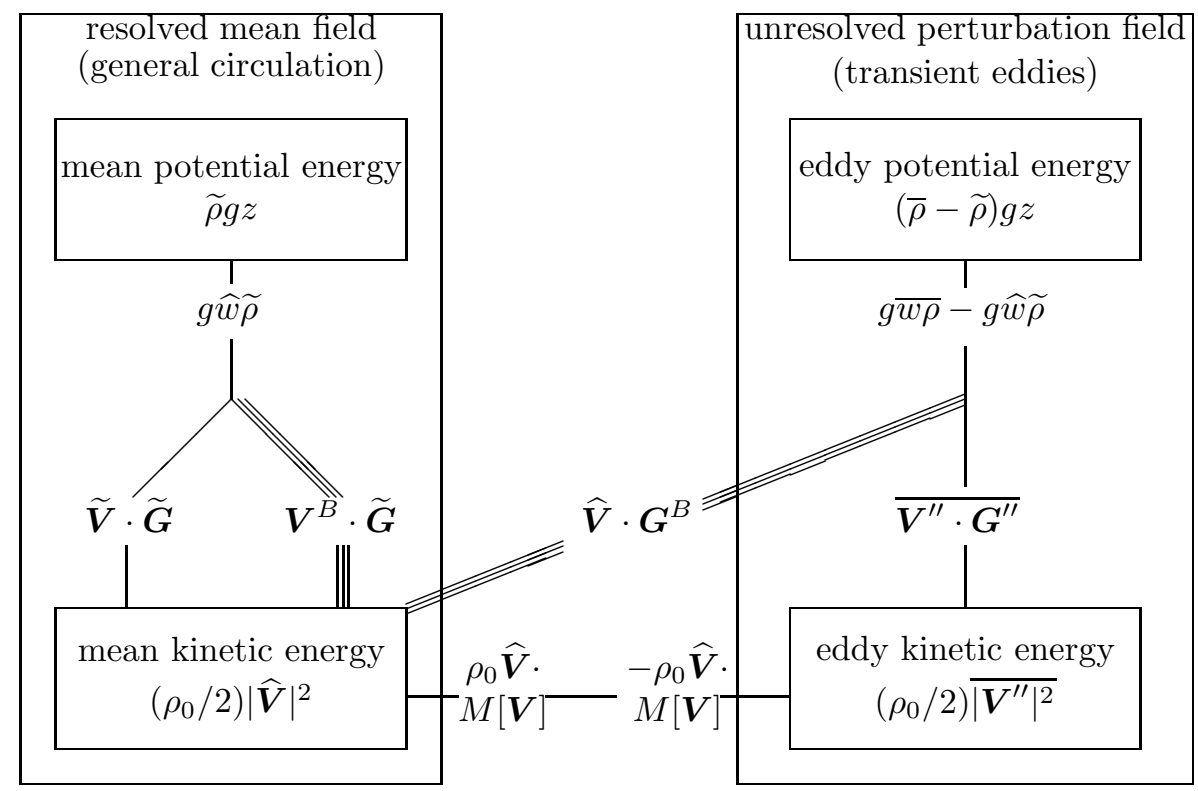

Fig. 2. The energy diagram for the thickness-weighted-mean formulation of an adiabatic hydrostatic Boussinesq fluid. Energy budgets are evaluated after taking the volume integral in a closed domain $\Omega$ based on Eqs. (13-16), (18), and (20). Details are in Sect. 3.4.

the mean and perturbation fields but because the TEM energy equations assume geostrophy in some terms in the primitive equations. The major difference between the present formulation (Bleck, 1985; Iwasaki, 2001; Jacobson and Aiki, 2006) and those of both Lorenz and the TEM theory comes from the modified definition for the mean and eddy kinetic energies, including the momentum equation being written for the total transport velocity and the modified pressure term incorporating the layer-thickness form drag. On the other hand, the definition of the mean and eddy potential energies given in this paper is similar to those in the classical Lorenz diagram and in the TEM diagram except for the direction of averaging.

Another concern is the boundary condition. The application of the TEM energy cycle to the Eady problem described in Sect. 5a of Plumb (1983) is complicated by the nonphysical energy fluxes through the top and bottom boundaries: the domain integrated fluxes divergences, such as $\nabla \cdot \boldsymbol{F}_{*}\left(K_{M}\right)$ and $\nabla \cdot \boldsymbol{F}_{*}\left(K_{E}\right)$, are nonzero. These energy fluxes in Plumb (1983) correspond to the pressure fluxes in the present study: we show that the volume integral of the pressure flux divergence clearly vanishes in the mean field, Eq. (15), and in the eddy field, Eq. (20).

To summarize, the adiabatic mean formulation presented here provides several unique features: (i) the total transport velocity $\widehat{\boldsymbol{U}}$ has no component normal to solid boundaries (Sect. 2.2), (ii) velocity $\boldsymbol{V}^{B}$ of the eddy-induced overturning circulation is included in the mean kinetic energy $\left(\rho_{0} / 2\right)|\widehat{\boldsymbol{V}}|^{2}$, (iii) geostrophic balance is not used in closing the energy diagram, and (iv) the presence of density surface perturbations (i.e., eddy potential energy) is directly related to the form drag working on the basic geostrophic currents: this replaces the route via the eddy kinetic energy in the TEM theory.

\subsection{Assumptions}

Additional results may be obtained if further assumptions are made. For example, if the basic current is largely geostrophic $\left(\rho_{0} f z \times \widetilde{\boldsymbol{V}} \simeq \widetilde{\boldsymbol{G}}\right)$ and the form drag is approximated as $\boldsymbol{G}^{B} \simeq \rho_{0} f \boldsymbol{z} \times \boldsymbol{V}^{B}$ in middle and high latitudes as is often done (Rhines, 1979; Greatbatch, 1998), the input from the potential energy balances the output to the unresolved field (this is indicated by the triple line in Fig. 2):

$$
\begin{aligned}
\widehat{\boldsymbol{V}} \cdot \boldsymbol{G}^{B} & \simeq\left(\tilde{\boldsymbol{V}}+\boldsymbol{V}^{B}\right) \cdot\left(\rho_{0} f \boldsymbol{z} \times \boldsymbol{V}^{B}\right) \\
& =\widetilde{\boldsymbol{V}} \cdot\left(\rho_{0} f \boldsymbol{z} \times \boldsymbol{V}^{B}\right) \\
& =-\boldsymbol{V}^{B} \cdot\left(\rho_{0} f z \times \tilde{\boldsymbol{V}}\right) \\
& \simeq-\boldsymbol{V}^{B} \cdot \widetilde{\boldsymbol{G}} .
\end{aligned}
$$

A small exchange due to the barotropic component of the form drag will be ignored if we consider a form $\operatorname{drag} \boldsymbol{G}^{B}$ that causes no net force in each vertical column (i.e., redistributing momentum only in the vertical direction). Equation (22) implies that no energy accumulates in the mean kinetic energy, which in turn indicates that the layer-thickness form drag approach in OGCMs produces results similar to those given by the extra advection schemes (discussed in Sect. 4 of Gent et al., 1995).

Nevertheless, the use of Eqs. (1) and (2) in OGCMs may result in barotropic currents and interactions with the bot- 


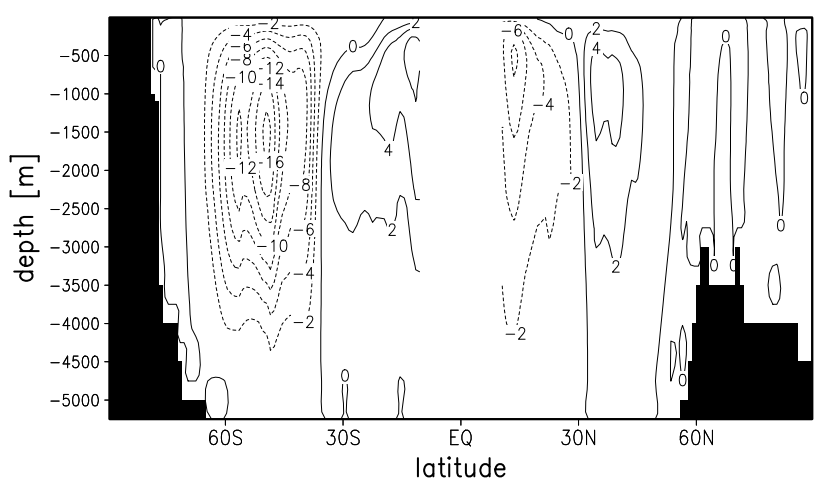

Fig. 3. Zonal integral of the meridional overturning stream function $\Psi^{y}=-\int_{-h}^{z} v^{B} d z$ (with units of $10^{6} \mathrm{~m}^{3} / \mathrm{s}$ ) in the global ocean, for the parameterized velocity in Eq. (24) with $C^{\prime}=0.02$. The baroclinic velocity $\widetilde{\boldsymbol{V}}_{b c}$ of the basic geostrophic current was calculated from the World Ocean Atlas. The solid (dashed) contours denote positive (negative) values.

tom topography that differ somewhat from those in the extra advection schemes, since the upper-layer momentum is gradually transferred down to the bottom by the form drag parameterization. Greatbatch and Li (2000) have reported that a three-dimensional simulation adopting the momentum approach is successful in showing anticyclonic mean flow around a seamount. Aiki et al. (2004) have demonstrated that relaxing the thermal wind balance of the basic field allows for barotropic currents to be present (see their Fig. 3). In the present energy cycle (Fig. 2), the fraction of the mean potential energy that is extracted by the eddy-induced overturning is able to interact with the mean kinetic energy before cascading to the unresolved field, in contrast to the extra advection schemes (based on the Lorenz diagram) that remove the mean potential energy directly.

\section{Work of the layer-thickness form drag}

It is of great interest to quantify the work of layer-thickness form drag in the world's oceans. Below we derive an indirect estimate of the energy conversion rate; realistic analyses of field measurements and numerical simulations are devoted to a later study.

\subsection{Scaling}

Greatbatch (1998) suggested that the layer-thickness form drag can be parameterized by Fickian diffusion that transfers the geostrophic momentum in the vertical direction (cf. Ferreira et al., 2005). In considering a similar form drag, Aiki et al. (2004) incorporated Rayleigh damping in the baroclinic component of the isopycnal mean velocity $\widetilde{\boldsymbol{V}}_{b c}$ :

$\boldsymbol{G}^{B} / \rho_{0}=-C^{\prime}|f| \widetilde{\boldsymbol{V}}_{b c}$,

where $C^{\prime}(>0)$ is a nondimensional constant. A series of preliminary numerical experiments were reported in Aiki et al. (2004), in which an arbitrary setting of $C^{\prime}=0.3$ was used with little justification for the rate of the overturning circulation. An appropriate value of $C^{\prime}$ that gives a realistic overturning circulation in the world's oceans is examined below.

Here we compute the form $\operatorname{drag}, \boldsymbol{G}^{B}$, starting with existing information about the eddy-induced velocity $\boldsymbol{V}^{B}$. Equation (23) with the form-drag approximation $\boldsymbol{G}^{B} \simeq \rho_{0} f \boldsymbol{z} \times \boldsymbol{V}^{B}$ (Sect. 3.6) yields the following eddy-induced extra velocity:

$\boldsymbol{V}^{B} \simeq C^{\prime} \frac{f}{|f|} z \times \widetilde{\boldsymbol{V}}_{b c}$.

The basic current of the velocity, $\widetilde{\boldsymbol{V}}_{b c}$, is assumed to be in thermal wind balance with the climatological density field of the World Ocean Atlas 2001 (Conkright et al., 2002), at least away from the equator. As plotted in Fig. 3 for the meridional overturning stream function, there are four distinct cells: (i) in the Southern Ocean associated with the instability of the Antarctic Circumpolar Current, (ii, iii) in the equatorial flanks that tend to lower the upwelling thermocline, and (iv) in the northern mid-latitudes corresponding to overturning cells of the Gulf Stream and the Kuroshio. We set $C^{\prime}=0.02$ so that the overturning rate in the Southern Ocean, which is $16 \times 10^{6} \mathrm{~m}^{3} / \mathrm{s}$ in Fig. 3, becomes as high as that in Figs. 6 and 7 of Gent et al. (1995). Although the eddy-induced circulation shown in Fig. 3 looks more intense between $30^{\circ} \mathrm{S}$ and $30^{\circ} \mathrm{N}$ (which will weaken the equatorial upwelling), the setting of $C^{\prime}=0.02$ for the simplified form drag in Eq. (23) can be regarded as corresponding to the standard horizontal diffusivity $\kappa=1000 \mathrm{~m}^{2} / \mathrm{s}$ in the parameterization of Gent and McWilliams (1990). If the magnitude of the bolus velocity is roughly $2 \%$ of the isopycnal mean velocity from Eq. (24) in the global ocean, estimates of the mean kinetic energy based on the thickness-weighted mean velocity and based on the unweighted mean velocity will yield very similar values, regardless of the physical meaning of the modified definitions of the mean and eddy kinetic energies (Sects. 3.1 and 3.3).

\subsection{Global conversion rate}

The quantity $\widehat{\boldsymbol{V}} \cdot \boldsymbol{G}^{B}$ in Eq. (22) is the work done by the form drag: momentum redistribution in the resolved mean field results in an energy cascade into the unresolved perturbation field. The quantity $-\boldsymbol{V}^{B} \cdot \widetilde{\boldsymbol{G}}$ is the depletion rate of the mean potential energy by the eddy-induced overturning. With the parameterization in Eqs. (23) and (24), these terms become $\widehat{\boldsymbol{V}} \cdot \boldsymbol{G}^{B} \simeq-\boldsymbol{V}^{B} \cdot \widetilde{\boldsymbol{G}}=-\rho_{0} C^{\prime}|f|\left|\widetilde{\boldsymbol{V}}_{b c}\right|^{2}$, which is 
negative in each vertical column. Figure 4 plots the column integral

$$
\int_{-h}^{0} \rho_{0} C^{\prime}|f|\left|\widetilde{\boldsymbol{V}}_{b c}\right|^{2} d z,
$$

for $C^{\prime}=0.02$ and velocity $\widetilde{\boldsymbol{V}}_{b c}$ from the World Ocean Atlas (Sect. 4.1). Energy extraction on the order of $10^{-2} \mathrm{~W} / \mathrm{m}^{2}$ is suggested in the regions of the Antarctic Circumpolar Current, the Gulf Stream, and the Kuroshio, which are indeed regions of enhanced eddy activity in the world's ocean currents. The global integral amounts to $0.41 \mathrm{TW}$ (1 TW=10 $\mathrm{W}$ ). Similar estimates using the Gent and McWilliams parameterization (Huang and Wang, 2003; Wunsh and Ferrari, 2004) give a range of 0.2-1.7 TW (which is reported to be very sensitive to the implementation technique). These values are comparable with the work done by the wind stress, which has been estimated at about $0.8 \mathrm{TW}$ (Wunsh, 1998). A global data assimilation for the layerthickness form drag, $\boldsymbol{G}^{B}$, in Eq. (2) by Ferreira et al. (2005) has confirmed that the work associated with the eddy stress in the ocean interior is close to that of the wind stress applied at the sea surface. The wind-induced Ekman transports can increase the mean potential energy through the energy channel $\widetilde{\boldsymbol{V}} \cdot \widetilde{\boldsymbol{G}}$ in Fig. 2, which makes the energy cycle of the (wind-driven) ocean circulation different from that of the (heat-driven) atmosphere circulation.

\section{Conclusions}

To understand the anisotropic mixing of momentum in a stratified fluid, we have investigated the residual effects of pressure perturbation (layer-thickness form drag) using the thickness-weighted temporal-averaged mean momentum and density equations (de Szoeke and Bennett, 1993; McDougall and McIntosh, 2001; Jacobson and Aiki, 2006). The layerthickness form drag connects the mean and eddy fields in a four-box energy diagram if the thickness-weighted mean velocity and the deviation from it are used for the definitions of the mean and eddy kinetic energies, respectively (Sects. 3.1 and 3.2). The adiabatic energy diagram in Sect. 3.4 should be consistent with the energy diagrams of layer models, TRM theory, and Iwasaki's atmospheric theory. The energy equations are obtained using an integral identity (called the "pileup rule") between cumulative sums of the Eulerian mean quantity and the thickness-weighted mean quantity in each vertical column (Sect. 2.2). The pile-up rule shows that the thickness-weighted mean velocity satisfies a no-normalflow boundary condition at the top and bottom of the ocean, which enables the volume budget of the pressure-flux divergence to be determined in the energy diagram (Sect. 3.5). The pile-up rule has also made it possible to rewrite the total kinetic energy based on the Eulerian mean, Eq. (8), in a

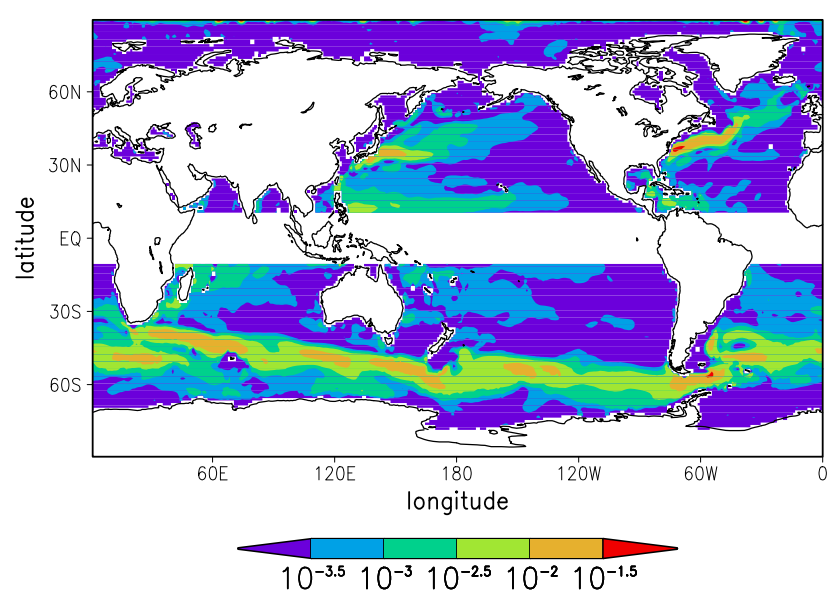

Fig. 4. A column integrated energy conversion rate $\rho_{0} C^{\prime}|f| \int_{-h}^{0}\left|\widetilde{\boldsymbol{V}}_{b c}\right|^{2} d z$, [W/ $\left.\mathrm{m}^{2}\right]$ in the world's oceans for the pressure drag in Eq. (23) with $C^{\prime}=0.02$. The baroclinic velocity $\widetilde{\boldsymbol{V}}_{b c}$ of the basic current was calculated from the World Ocean Atlas.

thickness-weighted form, Eq. (17). Necessary conditions for the derivation of the adiabatic energy diagram are summarized as follows:

(i) The total transport velocity has no component across the top and bottom boundaries (which represents an appropriate boundary condition for the pressure flux in the mean field).

(ii) The mean and eddy kinetic energies are defined using the total transport velocity and the deviation from it, respectively.

(iii) The momentum and density equations in the mean field are written for the total transport velocity and the mean height of density surfaces.

McDougall and McIntosh (2001) have contributed to prove condition (i), Bleck (1985) and Jacobson and Aiki (2006) have demonstrated conditions (ii) and (iii), and Iwasaki (2001) has explained all of the above three conditions. Regardless of the physical meaning of (ii), the scale analysis in the global ocean suggests that estimates of the mean kinetic energy based on the thickness-weighted mean velocity and based on the unweighted mean velocity will yield very similar values (Sect. 4.1). Under certain assumptions, the work of layer-thickness form drag in the global ocean circulation is suggested to be comparable to the work done by the wind forcing (Sect. 4.2). Direct analyses of high-resolution data in the Southern Ocean will serve to understand the relationship between the works done by the wind stress and the layer-thickness form drag (cf. Johnson and Bryden, 1989; Killworth and Nanneh, 1994; Rintoul et al., 2001). 
An application of the layer-thickness form drag is the parameterization of mesoscale eddies in the coarse-resolution ocean models used in climate studies (Greatbatch, 1998). The use of the thickness-weighted mean momentum Eq. (2) in OGCMs may result in barotropic currents and interactions with the bottom topography that differ somewhat from those in the extra advection schemes, since the adiabatic energy cycle enables the fraction of the mean potential energy to interact with the mean kinetic energy before cascading to the unresolved field (Sect. 3.6). Further studies should investigate the role of layer-thickness form drag in the ocean and atmosphere circulations.

Acknowledgements. The authors thank T. Iwasaki and T. Jacobson for useful discussions. This paper is improved by comments from R. Greatbatch and T. McDougall.

Edited by: S. M. Griffies

\section{References}

Aiki, H., Jacobson, T., and Yamagata, T.: Parameterizing ocean eddy transports from surface to bottom, Geophys. Res. Lett., 31, L19302, doi:10.1029/2004GL020703, 2004.

Andrews, R. G.: A finite-amplitude Eliassen-Palm theorem in isentropic coordinates, J. Atmos. Sci., 40, 1877-1883, 1983.

Andrews, R. G. and McIntyre, M. E.: Planetary waves in horizontal and vertical shear: the generalized Eliassen-Palm relation and the mean zonal acceleration, J. Atmos. Sci., 33, 2031-2053, 1976.

Andrews, R. G. and McIntyre, M. E.: An exact theory of nonlinear waves on a Lagrangian-mean flow, J. Fluid Mech., 89, 609-646, 1978.

Bleck, R.: On the conversion between mean and eddy components of potential and kinetic energy in isentropic and isopycnic coordinates, Dyn. Atmos. Oceans, 9, 17-37, 1985.

Böning, C. W. and Budich, R. G.: Eddy dynamics in a primitive equation model: sensitivity to horizontal resolution and friction, J. Phys. Oceanogr., 22, 361-381, 1992.

Canuto, V. M. and Dubovikov, M. S.: Dynamical model of mesoscales in z-coordinates, Ocean Modelling, 11, 123-166, 2006.

Charney, J. G.: The dynamics of long waves in a baroclinic westerly current, J. Meteorol., 4, 135-163, 1947.

Conkright, M. E., Locarnini, R. A., Garcia, H. E., O'Brien, T. D., Boyer, T. P., Stephens, C., and Antonov, J. I.: World Ocean Atlas 2001: Objective Analyses, Data Statistics, and Figures, CDROM Documentation, National Oceanographic Data Center, Silver Spring, MD, 2002.

Cushman-Roisin, B., Chassignet, E. P., and Tang, B.: Westward motion of mesoscale eddies, J. Phys. Oceanogr., 20, 758-768, 1990.

Danabasoglu, G. and McWilliams, J. C.: The role of mesoscale tracer transport in the global ocean circulation, Science, 264, 1123-1126, 1994.

de Szoeke, R. A. and Bennett, A. F.: Microstructure fluxes across density surfaces, J. Phys. Oceanogr., 23, 2254-2264, 1993.
Eady, E. T.: Long waves and cyclone waves, Tellus, 1, 33-52, 1949.

Ferreira, D., Marshall, J., and Heimbach, P.: Estimating eddy stresses by fitting dynamics to observations using a residualmean ocean circulation model and its adjoint, J. Phys. Oceanogr., 35, 1891-1910, 2005.

Gent, P. R. and McWilliams, J. C.: Isopycnal mixing in ocean circulation models, J. Phys. Oceanogr., 20, 150-155, 1990.

Gent, P. R., Willebrand, J., McDougall, T. J., and McWilliams, J. C.: Parameterizing eddy-induced tracer transports in ocean circulation models, J. Phys. Oceanogr., 25, 463-474, 1995.

Greatbatch, R. J.: Exploring the relationship between eddy-induced transport velocity, vertical momentum transfer, and the isopycnal flux of potential vorticity, J. Phys. Oceanogr., 28, 422-432, 1998.

Greatbatch, R. J. and Li, G.: Alongslope mean flow and associated upslope bolus flux of tracer in a parameterization of mesoscale turbulence, Deep-Sea Res., 47, 709-735, 2000.

Greatbatch, R. J. and McDougall, T. J.: The non-Boussinesq temporal residual mean, J. Phys. Oceanogr., 33, 1231-1239, 2003.

Griffies, S. M.: Fundamentals of Ocean Climate Models, Princeton University Press, 2004.

Holton, J. R.: An Introduction to Dynamic Meteorology, 3rd ed., Academic Press, 1992.

Huang, R. X. and Wang, W.: Gravitational potential energy sinks in the oceans, in: Proceedings of the 13th "Aha Huliko" a Hawaiian Winter Workshop, pp. 239-247, http://www.soest.hawaii.edu/ PubServices/AhaHulikoa.html, 2003.

Iwasaki, T.: Atmospheric energy cycle viewed from wave-meanflow interaction and Lagrangian mean circulation, J. Atmos. Sci., 58, 3036-3052, 2001.

Jacobson, T. and Aiki, H.: An exact energy for TRM theory, J. Phys. Oceanogr., 36, 558-564, 2006.

Johnson, G. C. and Bryden, H. L.: On the size of the Antarctic Circumpolar Current, Deep-Sea Res., 36, 39-53, 1989.

Kanzawa, H.: Quasi-geostrophic energetics based on a transformed Eulerian equation with application to wave-zonal flow interaction problems, J. Meteorol. Soc. Japan, 62, 36-51, 1984.

Killworth, P. D.: On the parameterization of eddy transfer. Part I: Theory, J. Mar. Res., 55, 1171-1197, 1997.

Killworth, P. D. and Nanneh, M. M.: Isopycnal momentum budget of the Antarctic Circumpolar Current in the Fine Resolution Antarctic Model, J. Phys. Oceanogr., 24, 1201-1223, 1994.

Killworth, P. D.: Boundary conditions on quasi-Stokes velocities in parameterizations, J. Phys. Oceanogr., 31, 1132-1155, 2001.

Kuo, A., Plumb, R. A., and Marshall, J.: Transformed Eulerianmean teory. Part II: Potential vorticity homogenization and the equilibrium of a wind- and buoyancy-driven zonal flow, J. Phys. Oceanogr., 35, 175-187, 2005.

Kushner, P. J. and Held, I. M.: Potential vorticity thickness fluxes and wave mean flow interaction, J. Atmos. Sci., 58, 948-958, 1999.

Lee, M. M. and Leach, H.: Eliassen-Palm flux and eddy potential vorticity flux for a nonquasigeostrophic time-mean flow, J. Phys. Oceanogr., 26, 1304-1319, 1996.

Lorenz, E. N.: Available potential energy and the maintenance of the general circulation, Tellus, 2, 157-167, 1955.

McDougall, T. J.: Three-dimensional residual-mean theory, in: Ocean Modelling and Parameterization, edited by Chassignet, 
E. P. and Verron, J., chap. 12, pp. 269-302, Kluwer Academic Publishers, 1998.

McDougall, T. J. and McIntosh, P. C.: The temporal-residual-mean velocity. Part I: Derivation and the scalar conservation equations, J. Phys. Oceanogr., 26, 2653-2665, 1996.

McDougall, T. J. and McIntosh, P. C.: The temporal-residual-mean velocity. Part II: Isopycnal interpretation and the tracer and momentum equations, J. Phys. Oceanogr., 30, 1222-1246, 2001.

Plumb, R. A.: A new look at the energy cycle, J. Atmos. Sci., 40, 1669-1688, 1983.

Plumb, R. A. and Ferrari, R.: Transformed Eulerian-mean theory. Part I: Nonquasigeostrophic theory for eddies on a zonal-mean flow, J. Phys. Oceanogr., 35, 165-174, 2005.
Rintoul, S. R., Hughes, C. W., and Olbers, D.: The Antarctic Circumploar Current system, in: Ocean Circulation \& Climate, edited by: Siedler, G., Church, J., and Gould, J., chap. 4.6, pp. 271-302, Academic Press, 2001.

Treguier, A. M., Held, I. M., and Larichev, V. D.: Parameterization of quasigeostrophic eddies in primitive equation ocean models, J. Phys. Oceanogr., 27, 567-580, 1997.

Wunsch, C.: The work done by the wind on the oceanic general circlulation, J. Phys. Oceanogr., 28, 2332-2340, 1998.

Wunsch, C. and Ferrari, R.: Vertical mixing, energy, and the general circulation of the oceans, Annu. Rev. Fluid Mech., 36, 281-314, 2004. 